\title{
ÍNDICES PLAQUETÁRIOS EM INDIVÍDUOS COM DOENÇA HEPÁTICA ALCOÓLICA CRÓNICA
}

\author{
Ana Cláudia COSTA ${ }^{1,2}$, Barbara RIBEIRO² e Elísio COSTA ${ }^{1}$
}

\begin{abstract}
RESUMO - Objetivo - Detectar alterações nos índices plaquetários em indivíduos com doença hepática alcoólica crónica e trombocitopenia e determinar o seu grau de correlação com outros parâmetros hematológicos. Métodos - Foram estudados um total de 65 indivíduos, divididos em dois grupos: controlos $(n=35)$ e com doença hepática alcoólica crónica e trombocitopenia $(n=30)$. O grupo controlo foi emparelhado por idade e sexo com o grupo de doentes. A todos eles, foi efectuado hemograma completo, incluindo índices plaquetários. Resultados - Os doentes com doença hepática alcoólica crónica apresentaram contagem de eritrócitos, leucócitos e plaquetas significativamente inferiores aos encontrados na população controlo. O mesmo se verificou com os valores de hemoglobina, hematócrito e valores absolutos de linfócitos e neutrófilos. O volume globular médio, a hemoglobina globular média e o RDW ("red cell distribution width") revelaram-se significativamente superiores no grupo de doentes. Relativamente aos índices plaquetários, foi encontrado aumento estatisticamente significativo do PDW ("platelet distribution width") e uma diminuição estatisticamente significativa no plaquetrócrito no grupo de doentes quando comparado com o grupo controlo. Em relação ao volume plaquetário médio não se encontraram diferenças estatisticamente significativas entre os dois grupos. Foi encontrada correlação estatisticamente significativa entre a contagem de plaquetas e os outros parâmetros hematológicos analisados. O plaquetrócito revelou-se o índice plaquetário que mais se relaciona com os restantes parâmetros hematológicos. Conclusão - Os doentes com doença hepática alcoólica crónica apresentam diminuição nas três linhas hematopoiéticas, possivelmente associada com o hiperesplenismo que estes doentes apresentam. Adicionalmente às alterações numéricas, encontraram-se alterações morfológicas a nível dos eritrócitos e plaquetas, demonstráveis pelos índices eritrocitários e plaquetários.
\end{abstract}

DESCRITORES - Plaquetas. Hepatopatias alcoólicas. Trombocitopenia.

\section{INTRODUÇÃO}

O consumo de álcool é aceite socialmente, mesmo quando consumido em doses muito elevadas. Por esta razão, o alcoolismo é actualmente um problema de saúde pública mundial, sendo doença encontrada em indivíduos de diferentes níveis socioeconómicos e de diferentes grupos étnicos, tendo repercussões negativas e consequências nefastas a nível orgânico, social e psicológico ${ }^{(2,4,9)}$. Uma das principais consequências orgânicas associadas com o alcoolismo é a doença hepática alcoólica crónica (DHAC), sendo o seu diagnóstico precoce difícil, uma vez que nem sempre é fácil fazer relação directa entre o consumo de álcool e a doença hepática ${ }^{(8,12)}$.

Estão descritos muitas alterações orgânicas associadas ao consumo excessivo de álcool, nomeadamente a nível da medula óssea. Estão descritas alterações nas linhas eritróide, granulocítica e megacariocítica, isoladamente ou concomitantemente, assim como alterações hematológicas a nível periférico, sendo as mais evidentes a anemia, a leucopenia, trombocitopenia e aumento no volume globular médio (VGM) $)^{(6,9)}$.

A trombocitopenia é um dos achados laboratoriais mais comuns na DHAC, estando habitualmente associada com hipertensão portal e a aumento da sequestração esplénica. A etiopatogenia desta trombocitopenia não está ainda totalmente esclarecida. Várias hipóteses têm vindo a ser levantadas e estudadas, nomeadamente, o aumento da sequestração esplénica, a produção inapropriada pela medula óssea, e diminuição da sobrevida das plaquetas em circulação ${ }^{(3,15,16)}$.

O objectivo deste estudo foi detectar alterações nos índices plaquetários em indivíduos com DHAC que desenvolveram trombocitopenia, e determinar o seu grau de correlação com outros parâmetros hematológicos.

\footnotetext{
1Departamento de Tecnologias de Diagnóstico e Terapêutica da Escola Superior de Saúde do Instituto Politécnico de Bragança. ${ }^{2}$ Serviço de Patologia Clínica do Hospital Distrital de Chaves, Bragança, Portugal.

Correspondência: Dr. Elísio Costa - Departamento das Ciências e Tecnologias da Saúde - Escola Superior de Saúde - Instituto Politécnico de Bragança - Avenida D. Afonso V - 5300-121 Bragança-Portugal. E-mail: elisio.costa@ipb.pt
} 


\section{MÉTODOS}

Foram incluídos neste trabalho todas as amostras que deram entrada no serviço de Patologia Clínica da Hospital Distrital de Chaves, Portugal, provenientes de doentes com DHAC que apresentassem trombocitopenia (contagem de plaquetas inferior a $\left.150 \times 10^{3} / \mu \mathrm{L}\right)$, durante um período de 10 meses. Em todos os casos, para além de se registar a idade e o sexo, foi efectuado hemograma completo, incluindo contagem de plaquetas e os índices plaquetários, utilizando o aparelho automático Cell Dyn3500 (Abbott Diagnostics, Califórnia, EUA). Foi incluído um grupo controlo, constituído por indivíduos emparelhados por idade e sexo, com o grupo de doentes. Estes indivíduos foram seleccionados na avaliação laboratorial pré-operatória e não apresentavam alterações nos parâmetros hematológicos, nem indicadores laboratoriais de infecção/inflamação.

No tratamento estatístico dos resultados foi utilizado o teste $t$ Student para comparação de médias e a correlação de Pearson para determinar o grau de correlação entre os diferentes parâmetros analisados. Foi considerado como significativo valores de $P$ inferiores a 0.05 .

\section{RESULTADOS}

Durante o período em que decorreu o estudo, foram seleccionados um total de 65 indivíduos, divididos em dois grupos: controlos $(\mathrm{n}=35)$ e com DHAC e trombocitopenia $(\mathrm{n}=30)$. A média de idades era de 49.7 anos (variando entre os 35 e os 74 anos) e a percentagem de indivíduos do sexo masculino era de $89 \%$. Não foi encontrada diferença estatisticamente significativa $(P>0.05)$, na idade e distribuição por sexos entre os dois grupos (controlo vs doentes). Os indivíduos com DHAC apresentavam evidência de hipertensão portal e esplenomegalia.
Os achados laboratoriais estão sumariados na Tabela 1. Os doentes com DHAC apresentam uma contagem de eritrócitos, hemoglobina, hematócrito e valores absolutos de leucócitos totais, linfócitos, neutrófilos e plaquetas significativamente inferiores aos encontrados na população controlo $(P<0.05)$. O VGM, a hemoglobina globular média e o RDW ("red cell distribution width") apresentam valores significativamente superiores $(P<0.05)$ no grupo de doentes.

Relativamente aos índices plaquetários foi encontrado no grupo de doentes um aumento estatisticamente significativo no PDW ("platelet distribution width") (19.01 $\pm 1.78(\%)$ vs $17.76 \pm 1.15(\%), P=0.004)$ (Figura 1 ) e diminuição estatisticamente significativa no plaquetrócito (PCT) $(0.082$ $\pm 0.031 \%$ vs $0.192 \pm 0.049 \%, P<0.005$ ) (Figura 2). Em relação ao volume plaquetário médio (VPM) não se encontraram diferenças estatisticamente significativas entre os dois grupos. Foi encontrada correlação estatisticamente significativa entre a contagem de plaquetas e os outros parâmetros hematológicos analisados (Tabela 2).

\section{DISCUSSÃO}

A trombocitopenia é reconhecidamente um achado comum associado com a ingestão de álcool. Contagem de plaquetas inferiores a $100 \times 10^{9} / \mathrm{L}$ ocorrem em aproximadamente $3 \%$ dos alcoólicos de longo tempo ${ }^{(2,4,9)}$. A etiologia desta trombocitopenia não está ainda completamente esclarecida, tendo vindo a ser associada com aumento da sequestração esplénica, produção inapropriada pela medula óssea, e diminuição da sobrevida das plaquetas em circulação ${ }^{(10,14)}$. Alguns estudos in vitro e experimentação animal têm demonstrado que doses elevadas de etanol são capazes de inibir o crescimento de colónias formadoras de megacariócitos e a diminuição dos níveis séricos de trombopoietina. Sendo o défice de produção a etiologia mais consensual para a trombocitopenia associada com o consumo de

TABELA 1. Resumo dos achados laboratoriais encontrados no grupo de doentes e na população controlo

\begin{tabular}{|c|c|c|c|}
\hline & Controlos & Doentes & Valor de $P$ \\
\hline Eritrócitos $\left(10^{6} / \mu \mathrm{L}\right)$ & $4,73 \pm 0,44$ & $3,27 \pm 0,80$ & $<0,001$ \\
\hline $\mathrm{Hb}(\mathrm{g} / \mathrm{dL})$ & $17,96 \pm 19,97$ & $11,30 \pm 2,49$ & 0,075 \\
\hline Hematócrito (\%) & $41,86 \pm 3,84$ & $31,18 \pm 7,05$ & $<0,001$ \\
\hline $\mathrm{MCV}(\mathrm{fL})$ & $88,47 \pm 2,90$ & $95,84 \pm 7,33$ & $<0,001$ \\
\hline $\mathrm{MCH}(\mathrm{pg})$ & $30,60 \pm 0,96$ & $34,87 \pm 3,60$ & $<0,001$ \\
\hline CHCM (g/dl) & $34,83 \pm 0,70$ & $35,53 \pm 3,78$ & 0,283 \\
\hline RDW (\%) & $13,95 \pm 0,789$ & $16,45 \pm 4,05$ & 0,001 \\
\hline Leucócitos $\left(10^{3} / \mu \mathrm{L}\right)$ & $7,39 \pm 2,919$ & $5,75 \pm 3,20$ & 0,039 \\
\hline Neutrófilos $\left(10^{3} / \mu \mathrm{L}\right)$ & $4,62 \pm 2,83$ & $3,65 \pm 2,62$ & 0,212 \\
\hline Linfócitos $\left(10^{3} / \mu \mathrm{L}\right)$ & $2,07 \pm 0,64$ & $1,3652 \pm 0,82$ & $<0,001$ \\
\hline Monócitos $\left(10^{3} / \mu \mathrm{L}\right)$ & $0,49 \pm 0,17$ & $0,60 \pm 0,35$ & 0,107 \\
\hline Eosinófilos $\left(10^{3} / \mu \mathrm{L}\right)$ & $0,15 \pm 0,10$ & $0,10 \pm 0,07$ & 0,036 \\
\hline Basófilos $\left(10^{3} / \mu \mathrm{L}\right)$ & $0,05 \pm 0,02$ & $0,08 \pm 0,15$ & 0,263 \\
\hline Plaquetas $\left(10^{3} / \mu \mathrm{L}\right)$ & $206,95 \pm 54,09$ & $74,36 \pm 34,76$ & $<0,001$ \\
\hline VPM (fL) & $9,39 \pm 1,142$ & $10,12 \pm 1,81$ & 0,065 \\
\hline PCT $(\%)$ & $0,19 \pm 0,04$ & $0,08 \pm 0,03$ & $<0,001$ \\
\hline PDW $(\%)$ & $17,75 \pm 1,15$ & $19,01 \pm 1,78$ & 0,002 \\
\hline
\end{tabular}


álcool ${ }^{(1,5,7,9,10,11,13,14)}$. Nos doentes desta série, em que o critério de inclusão era a presença de trombocitopenia, encontrou-se associada a presença de anemia e de leucopenia. A presença desta pancitopenia, associada à esplenomegalia e à hipertensão portal que estes doentes apresentam, sugere que a sua etiologia mais provável é a sequestração esplénica, já que todas as linhas

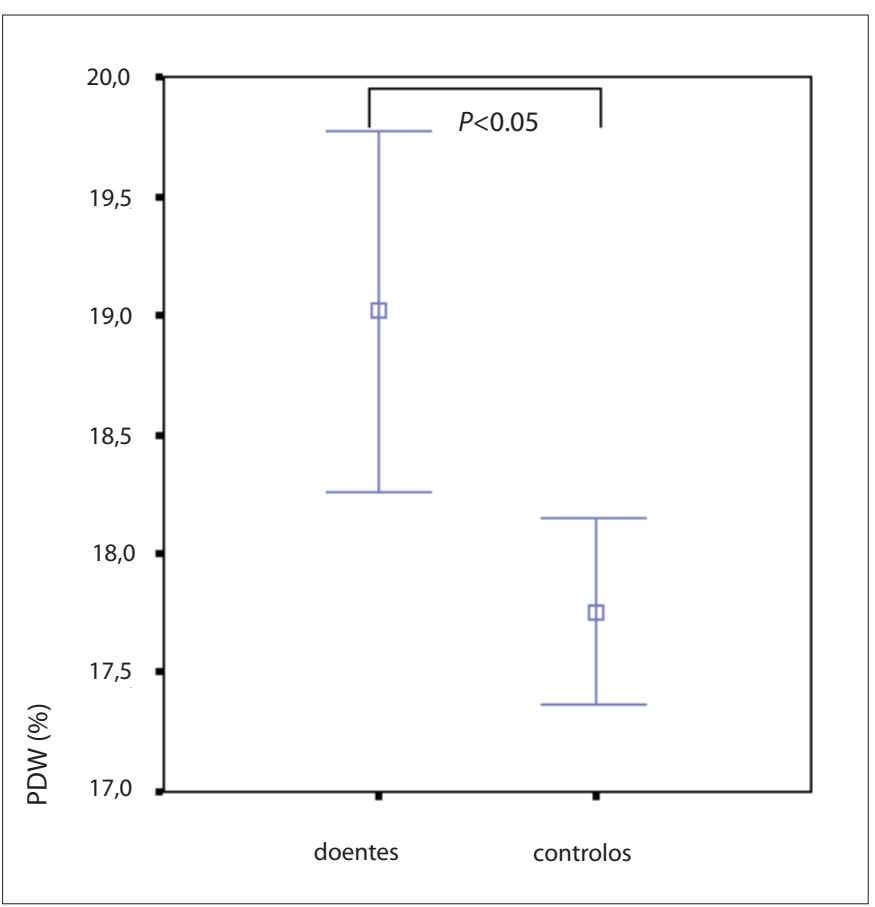

FIGURA 1. Aumento estatisticamente significativo no PDW encontrado no população de doentes celulares hematopoiéticas estão afectadas. No entanto, apenas o exame da medula óssea poderia dar informação mais definitiva sobre os efeitos do álcool na hematopoiese, o que não foi efectuada nestes doentes.

Os contadores hematológicos existentes, na maioria dos laboratórios clínicos, disponibilizam sem custos acrescidos,

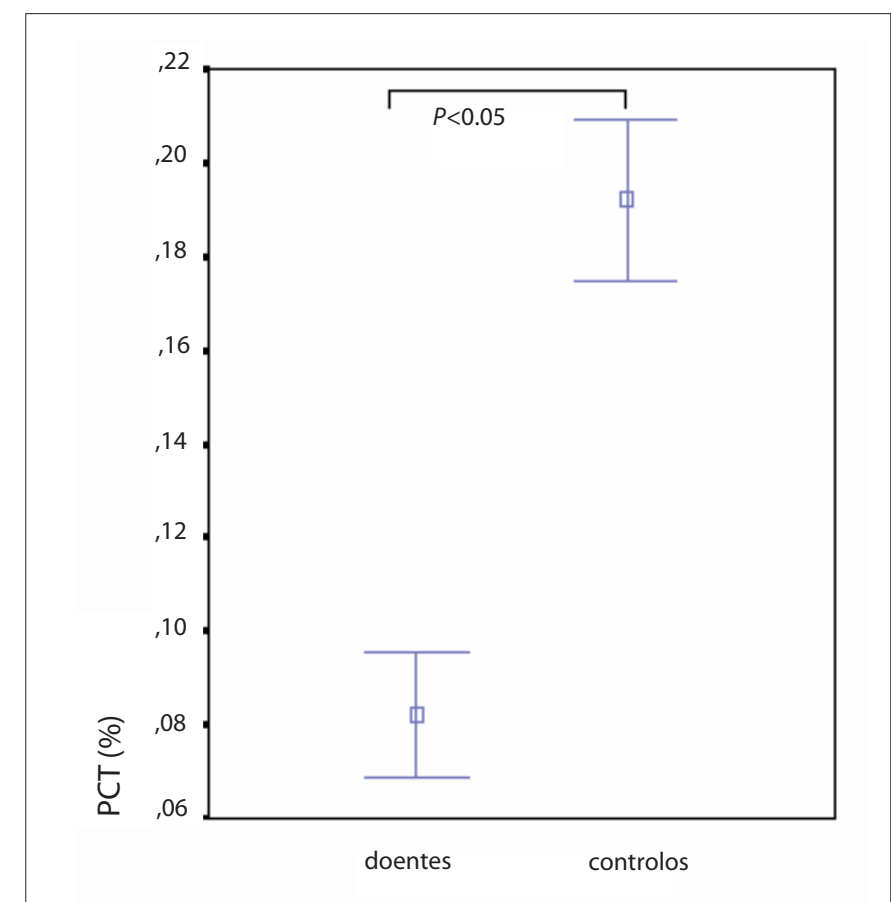

FIGURA 2. Diminuição estatisticamente significativa no PCT encontrado no população de doentes

TABELA 2. Correlação entre a contagem de plaquetas e os índices plaquetários entre si e com os outros parâmetros hematológicos

\begin{tabular}{|c|c|c|c|c|c|c|c|c|}
\hline & \multicolumn{2}{|c|}{$\begin{array}{l}\text { Plaquetas } \\
\left(10^{3} / \mu \mathrm{L}\right)\end{array}$} & \multicolumn{2}{|c|}{$\begin{array}{c}\text { VPM } \\
\text { (fL) }\end{array}$} & \multicolumn{2}{|c|}{$\begin{array}{c}\text { PCT } \\
(\%)\end{array}$} & \multicolumn{2}{|c|}{$\begin{array}{l}\text { PDW } \\
(\%)\end{array}$} \\
\hline & $\begin{array}{l}\text { Coeficiente de } \\
\text { correlação }\end{array}$ & Valor de $P$ & $\begin{array}{l}\text { Coeficiente de } \\
\text { correlação }\end{array}$ & Valor de $P$ & $\begin{array}{l}\text { Coeficiente de } \\
\text { correlação }\end{array}$ & Valor de $P$ & $\begin{array}{l}\text { Coeficiente de } \\
\text { correlação }\end{array}$ & Valor de $P$ \\
\hline Eritrócitos $\left(10^{6} / \mu \mathrm{L}\right)$ & 0,689 & $<0,001$ & $-0,239$ & 0,071 & 0,611 & $<0,001$ & $-0,220$ & 0,094 \\
\hline $\mathrm{Hb}(\mathrm{g} / \mathrm{dL})$ & 0,274 & 0,027 & $-0,018$ & 0,891 & 0,280 & 0,032 & $-0,096$ & 0,469 \\
\hline Hematocrito (\%) & 0,629 & $<0,001$ & $-0,239$ & 0,071 & 0,547 & $<0,001$ & $-0,173$ & 0,191 \\
\hline $\mathrm{MCV}(\mathrm{fL})$ & $-0,541$ & $<0,001$ & 0,087 & 0,518 & $-0,515$ & $<0,001$ & 0,247 & 0,059 \\
\hline $\mathrm{MCH}(\mathrm{pg})$ & $-0,610$ & $<0,001$ & 0,144 & 0,280 & $-0,582$ & $<0,001$ & 0,34 & 0,007 \\
\hline CHCM (g/dl) & $-0,168$ & 0,181 & 0,369 & 0,004 & $-0,067$ & 0,614 & 0,255 & 0,051 \\
\hline RDW (\%) & $-0,429$ & $<0,001$ & 0,106 & 0,427 & $-0,425$ & 0,001 & 0,197 & 0,134 \\
\hline Leucócitos $\left(10^{3} / \mu \mathrm{L}\right)$ & 0,390 & 0,001 & 0,058 & 0,664 & 0,337 & 0,009 & $-0,176$ & 0,184 \\
\hline Neutrófilos $\left(10^{3} / \mu \mathrm{L}\right)$ & 0,260 & 0,036 & 0,085 & 0,528 & 0,216 & 0,101 & $-0,154$ & 0,244 \\
\hline Linfócitos $\left(10^{3} / \mu \mathrm{L}\right)$ & 0,569 & $<0,001$ & $-0,142$ & 0,286 & 0,544 & $<0,001$ & $-0,177$ & 0,181 \\
\hline Monócitos $\left(10^{3} / \mu \mathrm{L}\right)$ & 0,031 & 0,808 & 0,196 & 0,140 & $-0,029$ & 0,829 & 0,038 & 0,777 \\
\hline Eosinófilos $\left(10^{3} / \mu \mathrm{L}\right)$ & 0,289 & 0,020 & 0,033 & 0,807 & 0,227 & 0,083 & 0,132 & 0,320 \\
\hline Basófilos $\left(10^{3} / \mu \mathrm{L}\right)$ & $-0,100$ & 0,429 & 0,416 & 0,001 & $-0,107$ & 0,419 & 0,104 & 0,435 \\
\hline Plaquetas $\left(10^{3} / \mu \mathrm{L}\right)$ & & & $-0,360$ & 0,005 & 0,956 & $<0,001$ & $-0,601$ & $<0,001$ \\
\hline VPM (fL) & & & & & $-0,145$ & 0,278 & 0,549 & $<0,001$ \\
\hline PCT $(\%)$ & & & & & & & $-0,466$ & $<0,001$ \\
\hline PDW (\%) & & & & & & & & \\
\hline
\end{tabular}


os resultados dos índices plaquetários que na maioria das vezes não são valorizados na prática clínica corrente. Uma das principais razões para isto acontecer está relacionada com o facto destes índices não terem sido ainda muito estudados, nomeadamente no que se refere à sua utilidade em termos de diagnóstico. Na literatura existem poucos trabalhos que tiram partido dos índices plaquetários no estudo de doentes com $\mathrm{DHAC}^{(11)}$. Este trabalho tenta efectuar essa abordagem, tendose demonstrado um aumento do PDW e uma diminuição do PCT nestes doentes. O primeiro parâmetro relaciona-se com anisocitose plaquetária e o segundo, está directamente relacionado com o volume que as plaquetas ocupam. Adicionalmente, foi encontrada boa correlação entre o número de plaquetas e índices plaquetários entre si, e também boa correlação entre a contagem de plaquetas e a maioria dos outros parâmetros hematológicos estudados. O PDW aumentado, encontrados nos doentes da presente casuística, poderá estar relacionada com o esforço que a medula óssea está a fazer no sentido de contrariar a trombocitopenia associada ao hiperesplenismo encontrado nestes doentes ou então, estar relacionada com os efeitos directos do álcool sobre as plaquetas. No entanto, mais estudos são necessários para que conclusões definitivas possam ser tiradas, nomeadamente estudos ao nível da medula óssea, estudos in vitro onde possam os efeitos directos do álcool nas células sanguíneas possam ser melhor caracterizados ou então, estudos em animais onde sejam criadas as condições in vivo.

Em conclusão, os doentes com DHAC apresentam alterações nas três linhas hematopoiéticas associadas com o hiperesplenismo. Adicionalmente, os eritrócitos e as plaquetas, apresentam evidência de alterações morfológicas, demonstráveis pelos respectivos índices, que poderão estar associadas com aumento da activa hematopoiética da medula óssea, resultante do hiperesplenismo ou por efeito directo do álcool.

Costa AC, Ribeiro B, Costa E. Platelet indices in chronic alcoholic liver disease patients with thrombocytopenia. Arq Gastroenterol. 2007;44(3):201-4.

ABSTRACT - Aim - To detect alterations in platelet indices in patients with chronic alcoholic liver disease and thrombocytopenia, and its correlation with other haematological parameters. Methods - We studied 65 individuals separated in two groups: controls $(\mathrm{n}=35)$ and chronic alcoholic liver disease patients with thrombocytopenia $(n=30)$. The control group was age and gender matched with patients group. In all, controls and patients, a haematological evaluation was done, including platelets indices. Results - In the patients group we found a low number of erythrocytes, leucocytes and platelet when we compare with controls. The same is true when we compare haemoglobin, hematocrit and absolute count of lymphocyte and neutrophil. The mean globular volume, mean globular haemoglobin and red cell distribution width where significantly higher in patients group. Platelet indices showed a statistical significant increased in platelet distribution width and decreased in platelet crit in the patient group. No differences where found on mean platelet volume between the two groups. Correlation between platelet number and other haematological parameters was found. Conclusion - Chronic alcoholic liver disease patients showed a decrease in all haematopoietic cell lines, probably associated with hypersplenism found in those patients. Additionally to the numeric alterations the erythrocyte and platelets showed morphologic alteration revelled by respective indices.

HEADINGS - Blood platelets. Liver diseases, alcoholic. Thrombocytopenia.

\section{REFERÊNCIAS}

1. Aiello A, Calapristi I, Freni MA, Fava A, Scarpignato EM, Spadaro A, Ferrau O. Assessment of portal hypertension in hepatic cirrhosis in relation to etiologic factors. Minerva Gastroenterol Dietol. 1993;39:1-5.

2. Andrade AG, Hirata E, Silveira MM, Bernick MA. Proposição de metodologia para avaliação da eficácia terapêutica em alcoólatras. J Bras Psiq. 1986;34:47-54.

3. Bashair FN, Teran JC, Mullen KD. Prevalence of peripheral blood cytopenias (hyperplenism) in patients with non-alcoholic chronic liver disease. Am J Gastroenterol. 2000;95:2936-9.

4. Beale RN, Bostrom JO, Taylor RF. Improved rapid methods for determination of iron content and binding capacity of serum. J Clin Pathol. 1962;15:156-60.

5. Bellis L, Castellacci R, Montagnese F, Festuccia F, Corvisieri P, Puoti C. Hepatic venous pressure gradient determination in patients with hepatitis $\mathrm{C}$ virus-related and alcoholic cirrhosis. Eur J Gastroenterol Hepatol. 2003;15:1085-9.

6. Conrad ME, Barton JC. Anemia and iron kinetics in alcoholism. Semin Hematol. 1980;17:149-63.

7. Eichner ER, Hillman RS. The evaluation of anemia in alcoholic patients. Am J Med. 1972;50:218-32.

8. Gluud C, Henriksen JH, Nielsen G. Prognostic indicators in alcoholic cirrhotic men. Hepatopatology. 1988;8:222.
9. Gualandro SFM. Efeitos diretos e indiretos do etanol sobre o eritron: estudo em alcoolistas sob regime ambulatorial [tese]. São Paulo: Faculdade de Medicina da Universidade de São Paulo; 1992.

10. Larkin EC, Watson-Williams EJ. Alcohol and blood. Med Clin North Am. 1984;68:105-20.

11. Latvala J, Parkkila S, Melkko J, Niemela O. Acetaldehyde adducts in blood and bone marrow of patients with ethanol-induced erythrocyte abnormalities. Mol Med. 2001;7:401-5.

12. Latvala J, Parkkila S, Niemela O. Excess alcohol consumption is common in patients with cytopenia: studies in blood and bone marrow cells. Alcohol Clin Exp Res. 2004;28:619-24.

13. Lieber CS. Medical disorders of alcoholism. Pathogenesis and treatment. Major Probl Intern Med. 1982;22:1-589.

14. Lindenbaum J, Hargrove RL. Trombocytopenia in alcoholics. Am Med. 1968;65:526-32.

15. Toghill P. The syndromes of splenic dysfunction. In: Bowdler AJ, editor. The spleen: structure, function and clinical significance. London: Chapman and Hall; 1990. p.209-32.

16. Toghill PJ, Green S. Splenic influences on the blood in chronic liver disease. Q J Med. 1979;48:613-25. 